\title{
MICROTOMOGRAFIA DE ALTA RESOLUÇÃO NO SETOR MINERAL
}

\author{
D. ULIANA ${ }^{1}$, H. KAHN ${ }^{2}$, R. CONTESSOTTO ${ }^{3}$ e J. L. ANTONIASSI ${ }^{4}$ \\ Depto. de Engenharia de Minas e de Petróleo - Laboratório de Caracterização Tecnológica (LCT) \\ E-mail: daniel@lct.poli.usp.br¹; henrique@lct.poli.usp.br²; renato@lct.poli.usp.br; juliana@lct.poli.usp.br
}

Artigo submetido em novembro/2013 e aceito em fevereiro/2014

DOI: http://dx.doi.org/10.15628/holos.2014.1775

\section{RESUMO}

A técnica de microscopia por tomografia de raios $X$ (MRX) oferece uma capacidade única para análise de associações, morfologia e liberação de múltiplas fases através da aquisição direta de imagens tridimensionais de alta resolução. Trata-se de uma técnica de análise 3D não invasiva atualmente utilizada no setor mineral para caracterização de carvão, minerais industriais e de base, além de metais preciosos, complementando técnicas de microscopia já existentes como MLA e QEMSCAN. A técnica já é empregada há algum tempo na indústria do petróleo para a caracterização de tamanho e distribuição de poros, permitindo simular o fluxo de óleo em rochas reservatório. Além disso, a caracterização de poros em 3D permite a simulação de fluxo em processos de lixiviação. Requerendo pouca ou nenhuma preparação da amostra, comparativamente às técnicas de microscopia bi ou unidimensionais, proporciona vantagens como a preparação muito mais ágil da amostra, aquisição direta de dados volumétricos, análise quantitativa em 3D e menor tempo de análise aliado a maior representatividade do material analisado. Particularmente em estudos de metais preciosos, tem-se um significativo aumento na probabilidade de detecção dos minerais de interesse. Recentes avanços com o emprego de dupla magnificação - projeção cônica aliada à utilização de lentes ópticas - possibilitam a aquisição de imagens com resolução submicrométrica com sensível melhoria no contraste entre as fases.

PALAVRAS-CHAVE: microtomografia, análise de imagens 3D, microscopia de raios X.

\section{HIGH RESOLUTION MICROTOMOGRAPHY ON MINING SECTOR}

\begin{abstract}
The technique of microscopy by X-ray computed tomography (XRM) offers a unique ability to analyze association, morphology and liberation of multiple phases through direct acquisition of high-resolution three-dimensional images. It is a non-invasive technique of 3D analysis currently used in the industry for characterization of coal, industrial and base minerals, besides precious metals, complementing existing microscopy techniques such as MLA and QEMSCAN. The technique is already used for some time in the petroleum industry to characterize the pore size distribution, allowing oil flow simulation in reservoir rocks. Furthermore, the $3 \mathrm{D}$ characterization of pores
\end{abstract}

allows flow simulations in leaching processes. Requiring little or no sample preparation, compared to two or one-dimensional microscopy techniques, it provides advantages such as much more agile sample preparation, direct acquisition of volumetric data, quantitative $3 \mathrm{D}$ analysis and less time analysis allied to major representativity of the analyzed material. Particularly in studies of precious metals there is a significant probability increase of interest minerals detection. Recent advances like dual magnification conical projection allied to optical lens - allow images acquisition with sub-micrometric resolution combined with considerable improvement in phases contrast.

KEYWORDS: microtomography; 3D image analysis; x-ray microscopy. 


\section{INTRODUÇÃO}

O conhecimento do minério e de sua variabilidade através da caracterização de partículas polifásicas é de suma importância desde a fase de exploração mineral até as etapas de operação da mina. De maneira geral, tais características são decisivas para definição de alternativas de processamento e dimensionamento de equipamentos para o beneficiamento mineral. A determinação das características microestruturais, tais como composições química e mineral, continuidade de fases, forma, associações minerais, espectro de liberação, área interfacial, dentre outras, é normalmente efetuada a partir de medições de partículas minerais em seções polidas, onde apenas uma parte das mesmas é exposta para a caracterização textural, o que leva a uma descrição incompleta da distribuição espacial das fases minerais (1D ou 2D - uni ou bidimensional). Além disso, algumas características são assumidas com base em informações texturais ou através de probabilidade geométrica, a fim de se realizar a correção estereológica a qual é limitada para situações de mais de duas fases (VIDELA et al., 2007).

Embora grandes avanços tenham sido conquistados na análise mineralógica automatizada sobre seções polidas, a análise de liberação mineral ainda é limitada pelas informações 1D ou 2D obtidas, o que leva à necessidade de transformação estereológica dos dados para uma informação mais precisa do espectro de liberação (SCHNEIDER et al., 1991). A técnica de microscopia por tomografia de raios $X$ de alta resolução (MRX) oferece uma capacidade única para análise quantitativa de sistemas de associação, morfologia e liberação de múltiplas fases através da aquisição direta de imagens tridimensionais de alta resolução. A caracterização microestrutural através de MRX proporciona vantagens como a preparação muito mais ágil da amostra, na aquisição direta de dados volumétricos, análise quantitativa em 3D, menor tempo de análise, etc. (MILLER e LIN, 2004).

\section{RAIOS X: DA DESCOBERTA AOS DIAS ATUAIS}

A descoberta dos raios X em 1895, pelo físico alemão Wilhelm Conrad Röntgen, proporcionou uma nova maneira de geração de imagem - a radiografia. A principal vantagem dos raios $X$ é a capacidade de penetrar em volumes espessos de matéria sem alterá-la, registrando-se então a diferença entre os materiais constituintes desde que possuam contraste natural (LIN et al., 2002). O contraste dos materiais numa radiografia se dá principalmente pela diferença de densidade, além da espessura em materiais de mesma densidade.

Após sua descoberta, a radiologia tornou-se uma área de especialização médica na década de 1900. Cinquenta anos depois, foi desenvolvido o amplificador de raios $\mathrm{X}$, o qual permitiu registrar as radiografias geradas em filmagens e apresentá-las em um monitor. A radiografia é amplamente utilizada para a inspeção de materiais em diversas aplicações até hoje. Nota-se, entretanto, que estruturas sobrepostas geram ambiguidade quanto à sua posição física na radiografia convencional. Este problema pode ser solucionado pelo emprego da tomografia (LIN, L. et al., op. cit.). 


\section{A TOMOGRAFIA DE RAIOS $X$}

O termo tomografia origina-se dos radicais gregos tomos, que significa parte de algo e grafein, o qual denota escrever ou registrar.

O princípio matemático da tomografia computadorizada foi desenvolvido no começo do século 20 por Radon (CNUDDE et al., 2006). A primeira aplicação comercial da tomografia computadorizada deu-se no final dos anos de 1960 e início de 1970, com o primeiro scanner por tomografia computadorizada, construído por Hounsfield (1973). Ele dividiu o Prêmio Nobel de Medicina, em 1979, com Allan Cormack, o qual desenvolveu alguns dos algoritmos matemáticos para a reconstrução tomográfica. Sua invenção mostrou que é possível calcular uma seção transversal com certa precisão, apesar de as projeções não satisfazerem estritamente os modelos teóricos fundamentais, além de os algoritmos de reconstrução serem de difícil implementação na época (KAK e SLANEY, 1987).

A reconstrução de projeções utilizando-se tomografia computadorizada (computerized tomography - CT) produz uma avaliação não invasiva da estrutura a partir de análises externas. $\mathrm{Na} \mathrm{CT}$, a radiação é transmitida ao longo de linhas retas através do objeto para um detector. A amostra é posicionada entre a fonte de emissão de raios $X$ e o detector, sendo então rotacionada para a aquisição de projeções em várias posições. Essas projeções são proporcionais à quantidade de radiação que chega ao detector, que varia de acordo com os distintos coeficientes de atenuação dos raios $X$ de cada material (LIN, L. et al., 2002). O processo de aquisição e reconstrução de imagens por tomografia computadorizada é ilustrado na Figura 1 (LANDIS e KEANE, 2010).

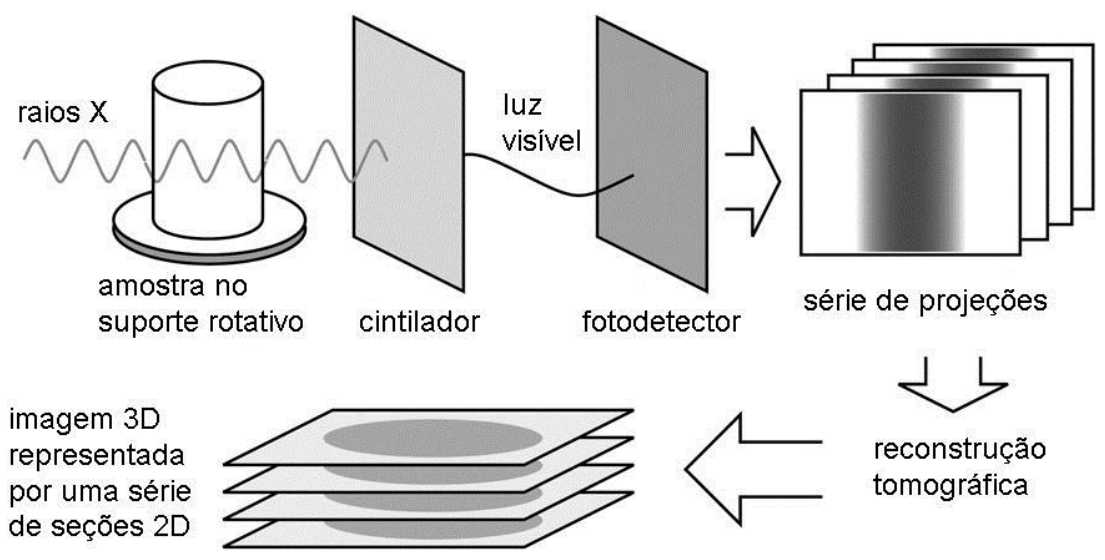

Figura 1 - Ilustração esquemática do processo de aquisição e reconstrução de imagens por tomografia computadorizada (LANDIS e KEANE, op. cit.)

\section{TIPOS DE TOMÓGRAFOS}

Desde 1972, os tomógrafos têm sido progressivamente aprimorados. A primeira geração consistia de um único arranjo de feixe de raios $X$ e detector. Na segunda geração, melhorou-se a qualidade de imagem pelo uso de múltiplos detectores em uma configuração de translação/rotação. Uma grande melhoria na velocidade foi obtida nos sistemas de terceira geração, os quais que se basearam na rotação helicoidal apenas da fonte de raios $\mathrm{X}$ e dos 
detectores em torno do objeto. Os equipamentos de quarta geração consistem de uma geometria de feixe helicoidal com rotação da fonte de raios $\mathrm{X}$ e detectores de alta eficiência dentro de um anel ao redor do objeto posicionado em uma mesa em movimento (WELLINGTON e VINEGAR, 1987).

Nas últimas décadas a medicina é, sem dúvida, o campo de aplicação que mais impulsionou a pesquisa e desenvolvimento da técnica de tomografia de raios X. Embora o princípio de funcionamento seja o mesmo para qualquer tipo de tomógrafo, os equipamentos utilizados na área médica apresentam arquitetura e mecanismo de funcionamento diferentes dos tomógrafos empregados em ciências dos materiais, na indústria mineral e em outras áreas não biológicas.

Em ambos os casos, o objeto a ser analisado deve ser posicionado entre a fonte de raios $\mathrm{X}$ e os detectores. Nos equipamentos de uso médico, o objeto (no caso o paciente) permanece em uma mesma posição, movimentando-se apenas na direção de um único eixo ortogonal à incidência do feixe de raios $X$, enquanto que o conjunto fonte-detectores é rotacionado em torno do objeto. Já no caso dos tomógrafos utilizados nas ciências não biológicas, o conjunto fontedetectores é estático e o objeto/amostra é rotacionado para incidência do feixe de raios $\mathrm{X}$ em várias posições. Exemplos dos dois tipos de equipamentos ilustrados na Figura 2.
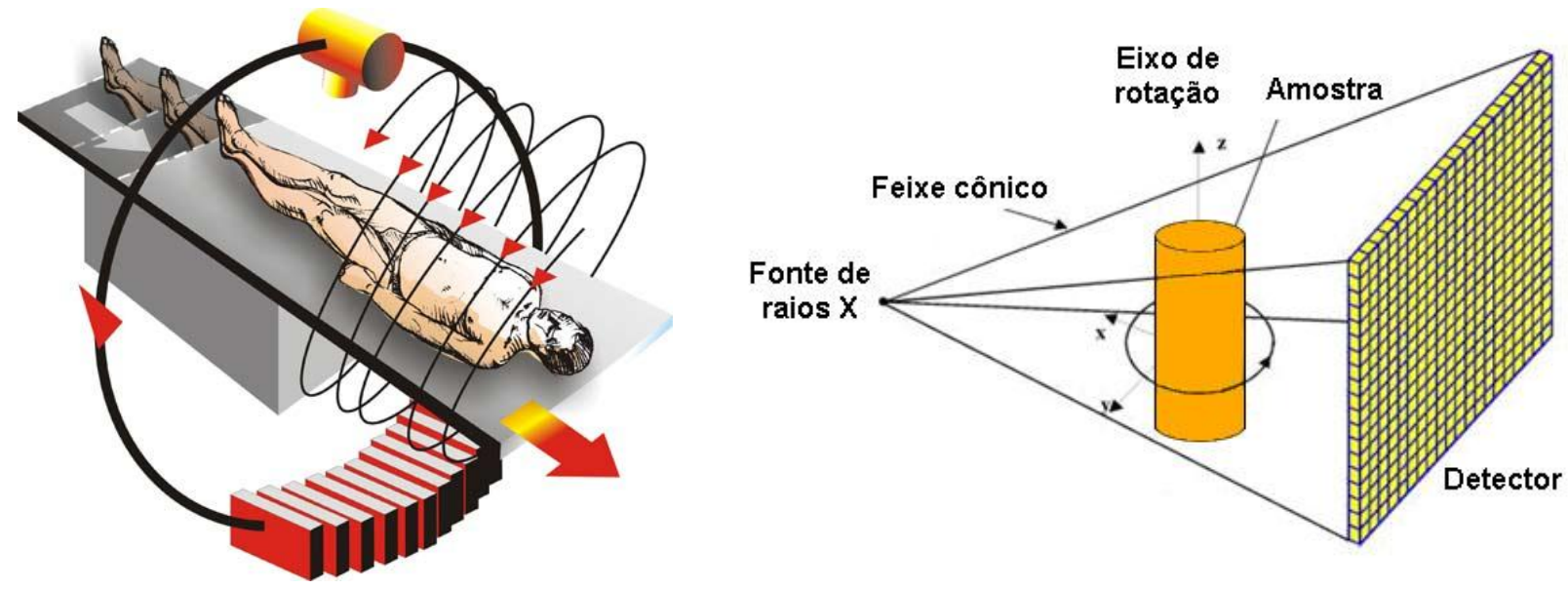

Figura 2 - Diferentes tipos de tomógrafos: tomógrafo empregado na área médica (esq.) e microtomógrafo utilizado para pesquisa mineral (dir.; (MILLER e LIN, 2009))

Sistemas médicos de CT por raios $\mathrm{X}$ possuem uma largura de feixe de alguns milímetros e uma fonte de energia de até $130 \mathrm{keV}$. Geralmente, tal sistema não seria adequado para a análise de liberação de partículas com dimensões de várias centenas de micrometros, o que seria de interesse para as operações de processamento mineral (LIN, L. et al., 2002). Com o advento da captura digital de imagens, a tomografia tornou-se a arte de reconstruir uma cópia tridimensional do objeto a partir fatias virtuais e está se tornando cada vez mais popular em toda uma gama de aplicações e escalas de tamanho (WITHERS, 2007).

A imagem digital por microscopia de raios $X$ de alta resolução foi estreada por Göttingen e Stony Brook na década de 1980 com fontes de radiação síncrotron. O constante progresso no aprimoramento da óptica e instrumentação das técnicas de raios $\mathrm{X}$ possibilitou atingir resoluções espaciais abaixo de 25 nanômetros, levando à proliferação mundial dessas técnicas. Como consequência, nos últimos anos, a ênfase da pesquisa foi mudando da instrumentação para as 
aplicações, criando uma demanda por sistemas de microscopia por raios $\mathrm{X}$ mais simples de operar e por processos automatizados de aquisição de dados e reconstrução de imagens em 3D (FESER, 2008).

\section{AVANÇOS RECENTES EM TOMOGRAFIA DE RAIOS X}

\subsection{Projeção cônica}

A geometria cônica do feixe de raios $X$ permite a reconstrução de um volume 3D isotrópico a partir da aquisição das projeções 2D com apenas uma rotação da amostra, sem a necessidade de translação mecânica e empilhamento de fatias sequenciais, tal como ocorre nos tomógrafos industriais (REIMANN et al., 1997; LIN e MILLER, 2002; WITHERS, 2007).

O emprego da projeção cônica permite que a distância entre a fonte emissora de raios $\mathrm{X} e$ a amostra possa ser bem pequena (até $<1 \mathrm{~mm}$ ). A imagem é normalmente obtida através da conversão de raios $X$ em luz visível por meio de um cintilador e projetada num dispositivo de carga acoplada (CCD). Quando a distância entre a fonte emissora e a amostra é grande, a resolução espacial é, muitas vezes, determinada pela resolução espacial do sistema de captura de imagem. Tanto os cintiladores, como a eficiência óptica e a difração da luz visível limitam a resolução espacial do sistema de captura de imagem de raios $X$ a cerca de $0,3 \mu \mathrm{m}$. Esta limitação pode ser minimizada através da utilização de uma geometria cônica de feixe, de modo a proporcionar uma imagem ampliada no detector (Figura 3). Neste caso, a resolução espacial é limitada pelo tamanho da fonte devido a efeitos de penumbra, com melhor resolução à medida que a distância fonte-amostra seja menor (REIMANN et al., op. cit.; LIN e MILLER, op. cit.;; WITHERS, op. cit.).

A resolução e o campo de visão são controlados pelas posições relativas entre fonteamostra (Dss) e amostra-detector (Dds) além das dimensões da fonte de raios $\mathrm{X}$ e resolução do detector. Esta configuração é a mais comum em tomógrafos de laboratório.

\subsection{Melhoria da óptica: combinação da projeção cônica com lentes}

Recentes avanços na óptica de raios $\mathrm{X}$ proporcionaram significativa melhoria na resolução dos sistemas de MRX com projeções pontuais, obtendo-se ganho de pelo menos uma ordem de grandeza em sistemas baseados em lentes de raios $X$. Microtomógrafos equipados com elementos ópticos como condensadores e lentes objetivas possibilitam atingir resoluções de imagem inferiores a 50 nanômetros, com significativa melhora de contraste (FESER, 2008; LAU et al., 2012). Um exemplo da geometria de um equipamento de laboratório com resolução espacial submicrométrica é apresentado na Figura 4. 


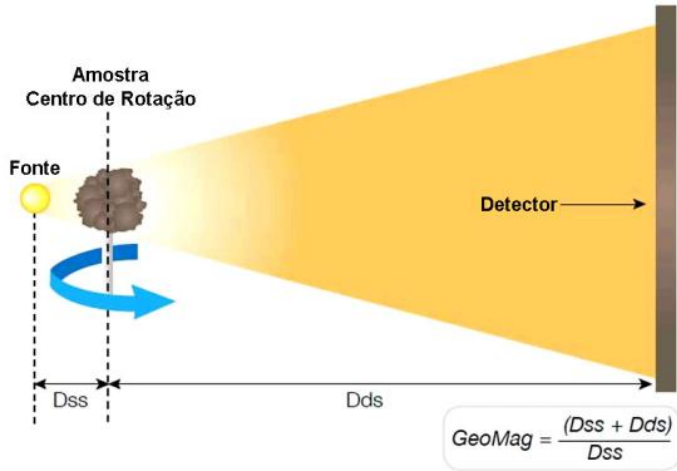

Figura 3 - Esquema de geometria de projeção cônica do feixe de raios $X$ (imagens XRADIA Inc.)

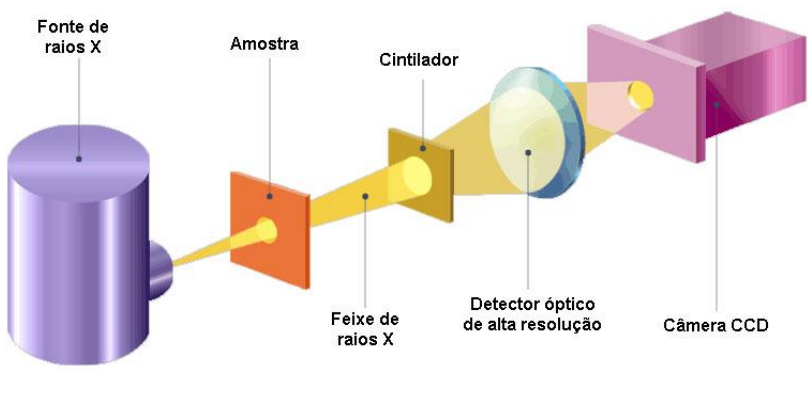

Figura 4 - Exemplo esquemático da geometria de equipamento com resolução espacial submicrométrica pela utilização de elementos ópticos (LAU et al., op. cit.).

A combinação da projeção cônica com a magnificação óptica (emprego de lentes, condensadores, etc.), permite que a resolução das imagens geradas seja independente do diâmetro do feixe de raios $X$, do tamanho da amostra e da distância entre a fonte e a amostra (LIN et al., 2012), com melhoria do contraste da imagem. Em outras palavras, um tomógrafo com essa combinação de recursos consiste em uma ferramenta bastante versátil em relação à resolução de imagem versus tamanho da amostra, permitindo assim a análise de microestruturas em diversas escalas de tamanho em uma vasta gama de Figura 5 com alta resolução e boa qualidade de contraste.

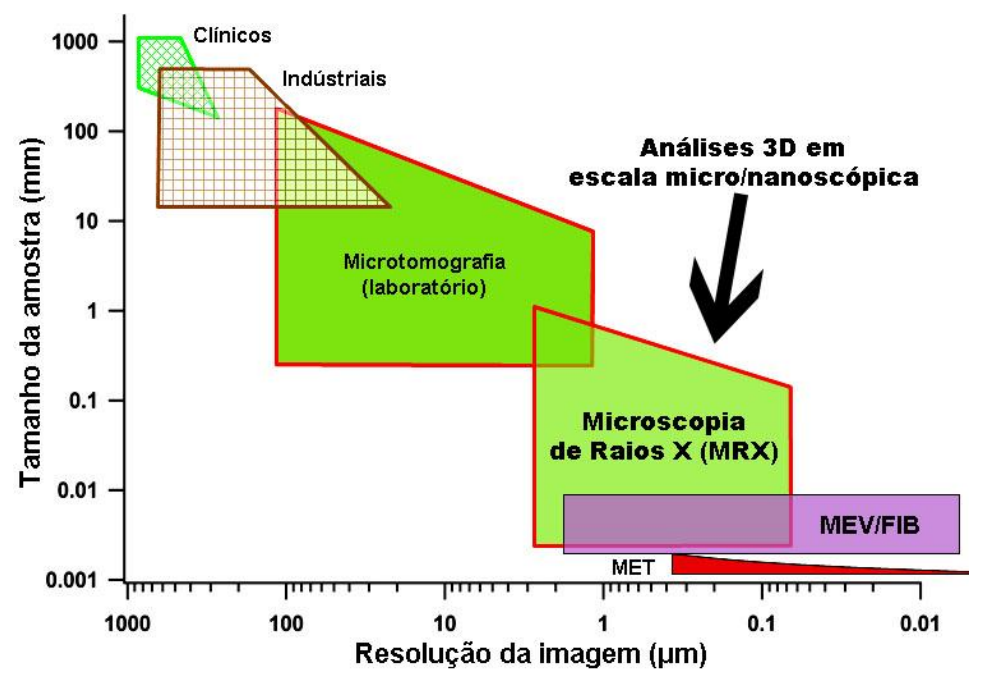

Figura 5 - Comparação de resolução de imagem e tamanho de amostra entre várias técnicas de caracterização 2D/3D (imagens XRADIA Inc.)

\section{ANÁLISES DE POROSIDADE}

A análise de porosidade é uma importante aplicação na ciência dos materiais, na caracterização de solos e outros tipos de materiais geológicos, etc. Os fluxos em meios porosos dependem das propriedades geométricas das partículas e seu empacotamento, assim como a estrutura de poros e a conectividade do espaço dos poros são características importantes que 
determinam o fluxo de fluido. A análise por MRX permite a digitalização em 3D da estrutura porosa e, aliada a métodos computacionais de simulação de fluxo, como o método de Boltzmann, torna-se uma importante ferramenta para a determinação do fluxo local a partir do qual as propriedades de macro-fluxo podem ser obtidas. Dessa maneira, é possível estudar os diferentes problemas de transporte de fluidos em escala de poros, como em rochas reservatório de petróleo ou em processo de lixiviação ácida (MEES et al., 2003; LIN et al., 2009; DHAWAN et al., 2012).

\section{APLICAÇÕES NA TECNOLOGIA MINERAL}

A MRX pode ser utilizada para a determinação em 3D dos danos causados às partículas após cominuição. As trincas e fraturas internas podem ser identificadas a partir da avaliação das imagens obtidas por MRX em alta resolução. Características das fraturas geradas, tais como quantidade, tamanho e forma podem ser medidas, calculando-se então a área da superfície específica das fraturas no produto moído, por meio de aplicações especialmente concebidas para a análise 3D. Com isso, pode-se definir o equipamento/mecanismo de cominuição mais adequado para determinado processo e averiguar em detalhe quais os níveis de danos causados às partículas em diferentes faixas granulométricas e o que isso irá impactar no processamento mineral (KODALI et al., 2011; LIN, MILLER et al., 2012).

Através da análise de imagens em 3D por microtomografia é possível estimar a recuperação de determinado elemento/mineral com base em dados reais de liberação em 3D e compará-los com a eficiência esperada para uma perfeita separação/concentração limitada apenas pela extensão da liberação. Neste sentido, a análise de liberação 3D por MRX é sempre preferível em relação à análise 2D, feita normalmente por microscopia em seções polidas, a qual tende a superestimar o grau de liberação (MILLER et al., 2009).

Outro exemplo de aplicação relevante para controle de processo é a análise de lavabilidade do carvão, que consiste em determinar o melhor resultado de separação a ser atingido. Dispondo-se de equipamento apropriado e devidamente calibrado, é possível monitorar o comportamento do material e avaliar o rendimento do processo através de uma curva de lavabilidade - peso específico/recuperação (LIN et al., 2000).

\subsection{Comparação/validação de resultados entre 2D e 3D (CT registration)}

A classificação na MRX é efetuada apenas com base no contraste de coeficientes de atenuação de raios $X$ de cada fase mineral (níveis de cinza), sendo esta diferenciação bem mais limitada que a dos sistemas por feixe de elétrons (p. ex. MLA e o QEMSCAN, os quais classificam os distintos minerais tanto com base na diferenciação de número atômico, como por composição química de cada mineral - espectros de EDS).

Neste sentido, fatias do volume de material analisado (testemunho de sondagem ou material particulado embutido em resina) por MRX podem ser submetidas à análise de imagens por feixe de elétrons (2D) para identificação e classificação das fases presentes. As imagens classificadas podem então serem registradas no software de classificação para extrapolação dos dados classificados de 2D para 3D (CT registration), através de algoritmos específicos para esta 
finalidade. Um exemplo desse processo é ilustrado na Erro! Fonte de referência não encontrada..

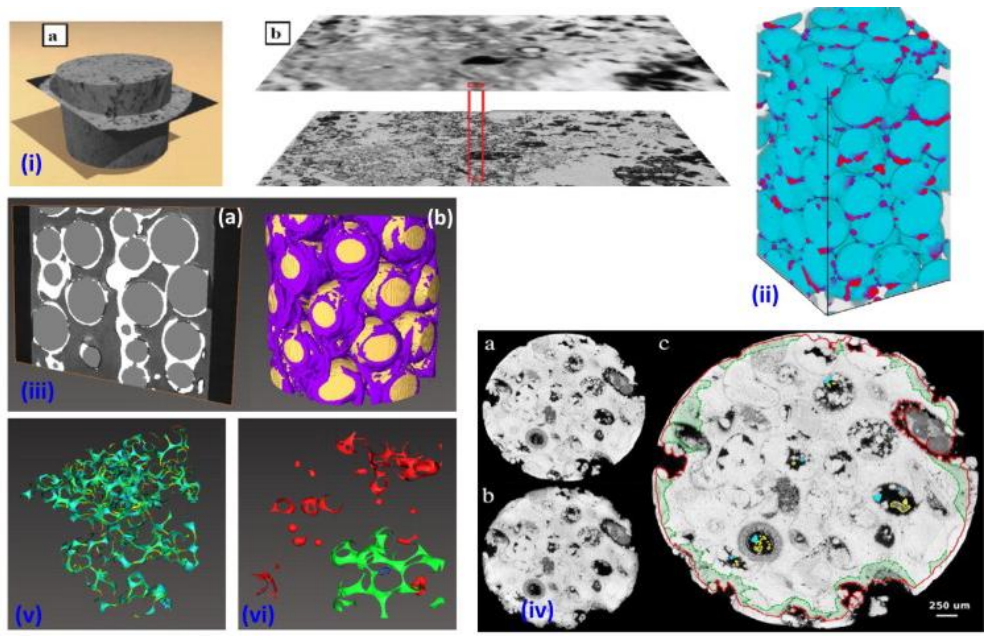

Figura 6 - Exemplo de registro de imagens 2D (MEV) para 3D (MRX): (i) fatias do volume (i-a) submetidas a análises no MEV (i-b); (ii) volume classificado por MRX (ii); (iii) melhor contraste de fases nas imagens de MEV (iii-

a) (a) e reclassificação do volume 3D (iii-b; CT registration); a estrutura porosa (iv) também é reconstruída (v) e segmentada em poros interconectados e isolados (vi) (WILDENSCHILD e SHEPPARD, 2013).

Este procedimento é efetuado para complementar a identificação e classificação de minerais indistinguíveis apenas por contraste de atenuação de raios $\mathrm{X}$.

\section{CONSIDERAÇÕES FINAIS}

A técnica de microscopia por tomografia de raios $X(M R X)$ vem se tornando uma ferramenta cada vez mais empregada no setor mineral por se tratar de uma técnica de análise não invasiva, de fácil preparação de amostra, boa versatilidade em relação ao tamanho de amostra analisada e elevada robustez estatística. Sucessivos aprimoramentos dos tomógrafos nas últimas décadas apontam para resoluções espaciais cada vez mais altas, alguns já na faixa de nanômetros. Os principais desafios hoje existentes são a melhoria de contraste nas imagens para maior eficiência na diferenciação entre as fases, principalmente para materiais de menor número atômico, além do aprimoramento de aplicações mais robustas para processamento das imagens.

\section{REFERÊNCIAS BIBLIOGRÁFICAS}

1. CNUDDE, V. et al. Recent progress in X-ray CT as a geosciences tool. Applied Geochemistry, v. 21, n. 5, p. 826-832, 2006.

2. DHAWAN, N. et al. Recent advances in the application of X-ray computed tomography in the analysis of heap leaching systems. Minerals Engineering, v. 35, p. 75-86, 2012.

3. FESER, M. Status and furure of commercial X-ray microscopes. In: 9th International Conference on X-Ray Microscopy, 2008. Zürich. Proceedings.Zürich: 2008.

4. KAK, A. C.; SLANEY, M. IEEE, New York: Principles of computerized tomographic imaging. 1987. $329 \mathrm{p}$.

5. KODALI, P. et al. Particle damage and exposure analysis in HPGR crushing of selected copper 
ores for column leaching. Minerals Engineering, v. 24, n. 13, p. 1478-1487, 2011.

6. LANDIS, E. N.; KEANE, D. T. X-ray microtomography. Materials Characterization, v. 61, n. 12, p. 1305-1316, 2010.

7. LAU, S. H.; MILLER, J. D.; LIN, C. L. 3D mineralogy, texture and damage analysis of multiphase mineral particles with a high contrast, submicron resolution X-ray tomography system. In: XXVI INTERNATIONAL MINERAL PROCESSING CONGRESS (IMPC), 2012. New Delhi. Proceedings.New Delhi: 2012. p. 2726-2736.

8. LIN et al. Cone beam X-ray microtomography: a new facility for three-dimensional analysis of multiphase materials. Minerals \& Metallurgical Processing, v. 19, n. 2, p. 7, 2002.

9. LIN, C.; MILLER, J. Cone beam X-ray microtomography: a new facility for three-dimensional analysis of multiphase materials. Minerals \& Metallurgical Processing, v. 19, n. 2, p. 65-71, 2002.

10. LIN, C. et al. Development of an on-line coal washability analysis system using X-ray computed tomography. Coal Perparation, v. 21, n. 4, p. 383-409, 2000.

11. LIN, C.; VIDELA, A.; MILLER, J. Advanced 3D multiphase flow simulation in porous media reconstructed from x-ray micro tomography using the He-Chen-Zhang Lattice Boltzmann model. 2009.

12. LIN, C. L.; MILLER, J. D.; HSIEH, C. H. Particle damage during HPGR breakage as described by specific surface area distribution of cracks in the crushed products. In: XXVI INTERNATIONAL MINERAL PROCESSING CONGRESS (IMPC), 2012. New Delhi. Proceedings.New Delhi: 2012. p. 3397-3410.

13. MEES, F. et al. Applications of X-ray computed tomography in the geosciences. Geological Society, London, Special Publications, v. 215, n. 1, p. 1-6, 2003.

14. MILLER, J. D.; LIN, C. High resolution X-ray micro CT (HRXMT) - Advances in 3D particle characterization for mineral processing operations. Recent Advances in Mineral Processing Plant Design, p. 48, 2009.

15. MILLER, J. D.; LIN, C. L. Three-dimensional analysis of particulates in mineral processing systems by cone beam X-ray microtomography. Minerals \& Metallurgical Processing, v. 21, n. 3, p. 113-124, 2004.

16. MILLER, J. D. et al. Liberation-limited grade/recovery curves from X-ray micro CT analysis of feed material for the evaluation of separation efficiency. International Journal of Mineral Processing, v. 93, n. 1, p. 48-53, 2009.

17. REIMANN, D. A. et al. A cone beam computed tomography system for true 3D imaging of specimens. Applied radiation and isotopes, v. 48, n. 10, p. 1433-1436, 1997.

18. SCHNEIDER, C. L. et al. Improved transformation technique for the prediction of liberation by a random fracture model. Powder Technology, v. 67, n. 1, p. 103-111, 1991.

19. VIDELA, A. R.; LIN, C. L.; MILLER, J. D. 3D characterization of individual multiphase particles in packed particle beds by X-ray microtomography (XMT). International Journal of Mineral Processing, v. 84, p. 321-326, 2007.

20. WELLINGTON, S.; VINEGAR, H. X-ray computerized tomography. Journal of Petroleum Technology, v. 39, n. 8, p. 885-898, 1987.

21. WITHERS, P. J. X-ray nanotomography. Materials today, v. 10, n. 12, p. 26-34, 2007. 